\title{
Chronicles of Oncology
}

Open Access | Mini-Review

\section{Risk of myocarditis in patients with cancer treated with immune checkpoint inhibitors.}

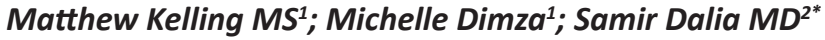 \\ ${ }^{1}$ Kansas City University of Medicine and Biosciences, Kansas City, Missouri, USA \\ ${ }^{2}$ Adjunct Clinical Assistant Professor of Hematology/Oncology, Kansas City University of Medicine and Biosciences, Joplin, Mis- \\ souri, USA
}

\author{
*Corresponding Author(s): Samir Dalia \\ Adjunct Clinical Assistant Professor of Hematology/ \\ Oncology, Kansas City University of Medicine and \\ Biosciences, Joplin, Missouri 64804, USA
}

Tel: 417-781-7722

Email: sdalia@gmail.com \& Samir.dalia@mercy.net

Received: March 30, 2018

Accepted: June 01, 2018

Published Online: June 07, 2018

Journal: Chronicles of Oncology

Publisher: MedDocs Publishers LLC

Online edition: http://meddocsonline.org/

Copyright: @ Dalia S (2018). This Article is distributed under the terms of Creative Commons Attribution 4.0 international License

\section{Introduction}

The field of oncology has changed immensely since the advent of immune checkpoint inhibitors (ICls). Cancers, such as advanced melanoma, previously carried a prognosis of 8-10 months, now patients have a chance at remission [1]. In certain patient populations with advanced non-small cell lung cancer (NSCLC), ICls are improving outcomes and becoming the standard of care [2]. The potency of these immunotherapy agents is due to their ability to disinhibit the body's immune response and unleash a vigorous antitumor response. Like any medication, these agents have associated side effects and due to their mechanism of action, they are associated with a broad range of auto-immune related adverse events (IRAEs). Some classes of ICls can cause low grade adverse events in $64-80 \%$ of patients
[3]. The most common IRAE's seen with ICls include fatigue, musculoskeletal pain, pruritus, nausea, vomiting, and diarrhea [4]. While most low grade toxicities are not severe and typically reversible, less frequent but more severe adverse events do occur. Recently in the literature case reports of $\mathrm{ICI}$ related cardiotoxicity have emerged. This review article will discuss incidence of cardiac events with ICls and the clinical management of these events should they arise.

\section{Immune checkpoint inhibitors}

ICls target two receptors involved in the regulation of $\mathrm{T}$ cell signaling and immune response, cytotoxic $T$ lymphocyte-associated antigen 4 (CTLA-4) and programmed cell death protein-1 (PD-1) $[5,6]$. These receptors function to attenuate the immune 
response and up regulate apoptosis of cytotoxic T cells. Cancer cells possess the uncanny ability to increase inhibitory signals to the host immune system through CTLA-4 and PD-1, thereby evading destruction by T cells. In 1995, a research team led by James Allison observed that a CTLA-4 blockade caused tumor necrosis in mice [7]. This discovery lead to the development of ipilimumab, a monoclonal antibody targeting CTLA-4 which is currently approved for treatment of advanced melanoma [8]. Subsequent research led to the development of monoclonal antibodies targeting PD-1 and its ligand, PD-L1. These ICls have gained approval for a broad spectrum of malignancies (see table 1), and new treatment strategies are currently being developed including those that combine ICls to improve treatment efficacy.

The first group of patients to demonstrate improved survival with $\mathrm{ICl}$ therapy were advanced melanoma patients treated with ipilimumab [8]. Advanced melanoma with traditional therapy has a median survival of 8-10 months, trials with ipilimumab have demonstrated 3-year survival rates of $22 \%$ [1]. Combining ipilimumab with nivolumab in this patient population has yielded even more impressive 3-year survival rates of $67 \%$ [11]. We are still in the infancy of $\mathrm{ICl}$ based therapy, so it is likely that we are only scratching the surface of their therapeutic potential.

\section{Immune related cardiotoxicity}

Due to the widespread effects of ICls on the immune system, almost any organ is a potential target for adverse events. The most common IRAEs include colitis, hepatitis, rash, and endocrinopathies [12]. The implications of ICls on the cardiovascular system are yet to be fully understood due to the relative rarity, lack of screening in clinical trials, and variable presentation of single case reports in the literature. The incidence of cardiotoxicity using a database of 20,594 patients treated with nivolum$a b$, or nivolumab plus ipilimumab reported $.06 \%$ in monotherapy and $.27 \%$ with combination therapy. However, due to lack of baseline EKGs and cardiac enzymes this is likely an under representation, and the potentially life-threatening consequences of these events have warranted more attention [13].

Johnson et al detailed two cases of fatal myocarditis and myositis in patients with melanoma being treated with combination ipilimumab and nivolumab. Postmortem pathologic analysis showed lymphocytic infiltrate in striated cardiac and skeletal muscle, and tumor tissue. Furthermore, the T cell receptor sequencing showed clonal expansion, suggesting there may be a shared antigen such as desmin or troponin that is triggering the T-cell-mediated autoimmune attack [13].

The types of cardiotoxicities described in case reports include myocarditis, myocardial fibrosis, cardiac arrest, heart failure including takosubo's cardiomyopathy, and conduction abnormalities $[3,14,15]$. Heinzerling et al. produced a multicenter case series of eight patients deemed likely to have experienced immune therapy-associated cardiotoxicity. Of these, 5 out of 8 patients had preexisting cardiac disease, although all were free of symptoms at time of therapy initiation and some stable for decades [3]. This raises the question of whether patients with previous cardiac disease should have increased screening prior to and throughout therapy. Reasonable suggestions include baseline EKG and cardiac enzymes prior to therapy. An echocardiogram may be useful as well, depending on the patient's risk factors and clinician's judgment [16].

\section{Clinical considerations and management}

Wang et al. has detailed an algorithm for management of both symptomatic and asymptomatic elevation of troponins during $\mathrm{ICl}$ therapy. For a significant troponin elevation in an asymptomatic patient, confirmation should be made with repeat assay followed by holding immunotherapy for 2 weeks. If normalization has occurred at this time, therapy can be restarted. If normalization does not occur after 2 weeks, a cardiology consult should be considered. For patients presenting with symptoms such as dyspnea, peripheral edema, chest pain, or palpitations with abnormal EKG and or cardiac enzymes, immunotherapy should be stopped and patient admitted to the hospital for further work up. Diagnosis can be made with cardiac MRI or myocardial biopsy [17].

The most important step in the management for $\mathrm{ICl}$ related myocarditis is rapid initiation of high dose glucocorticoids. Symptomatic management with diuretics may be used appropriately. If the patient is not improving, additional immunosuppressive agents may be required such as calcineurin inhibitors, cyclophosphamide, mycophenolate, and anti-thymocyte globulin [17].

With the proven mortality benefit of $\mathrm{ICl}$ agents for various malignancies, an important question clinicians are faced with is the safety of restarting immunotherapy following an IRAE. Some data suggests that IRAEs are associated with improved efficacy of the therapy, whereas this must be weighed against the risk for life threatening toxicities to develop. In general, the occurrence of an immune related adverse event, even requiring treatment with glucocorticoids, is not a contraindication for restarting immunotherapy. However, this does warrant heightened surveillance for recurrence and new toxicities during therapy. However, if a patient develops a high-grade life threatening toxicity, such as fulminant myocarditis, this is a contraindication to restarting therapy [12].

Notably, a recent case report describes a patient who was treated for $\mathrm{ICl}$ related myocarditis, but recurred shortly after reinitiating $\mathrm{ICI}$ therapy. This was a case of a 72-year-old male with prior comorbidities and extensive metastatic melanoma being treated with combination nivolumab and ipilimumab. After 4 infusions of combination $\mathrm{ICl}$ therapy the patient began to develop low grade IRAEs including autoimmune hyperthyroidism and autoimmune hypophysitis that were treated appropriately. After the $10^{\text {th }}$ nivolumab infusion the patient presented with dyspnea, edema, and weight gain. Echocardiogram showed reduced EF of $15 \%$ from baseline $48-50 \%$. With other etiologies ruled out, a cardiac biopsy confirmed autoimmune myocarditis, showing interstitial fibrosis and inflammatory infiltration with the absence of FOXP3 positive cells. Prednisolone and diuretic therapy were initiated and the patient improved, with EF reaching $35-40 \%$. At this time, restaging showed progressive disease, and the decision was made to restart single anti PD1 therapy (pembrolizumab) with close cardiac monitoring. After 2 weeks of therapy the patient developed dyspnea with minimal exertion, weight gain, and leg edema. He was treated again with steroids and diuretics and $\mathrm{ICl}$ was permanently stopped. The patient expired 2 months later following cardiac arrest [18].

\section{Conclusion}

Cardiotoxicity is an important complication of $\mathrm{ICl}$ therapy, and although quite rare, when it does occur it commonly proves fatal for the patient. Our literature highlights cases occurring 
with both anti-CTLA- 4 and anti-PD-1 agents, as well as those receiving monotherapy and combination therapy. With a growing number of approved indications as well as an ever-expanding number of clinical trials, it is not surprising that some of the rarer adverse effects of $\mathrm{ICls}$ have been unmasked. As the population of patients on ICls continues to grow it only increases the need for provider awareness of ICl related cardiotoxicity, which will lead to early recognition and appropriate clinical management. Clinicians should be suspicious of any patient receiving
$\mathrm{ICI}$ therapy displaying dyspnea, peripheral edema, chest pain, or palpitations with abnormal EKG and or cardiac enzymes; in these patients we support the treatment algorithm outlined by Wang et al. Hopefully further collaboration between cardiologists, oncologists, and researchers can lead to better strategies to prevent $\mathrm{ICl}$ induced cardiotoxicity as well as strategies to predict which patients are at increased risk by developing biomarkers and testing for genetic susceptibility.

Tables

Table 1: Current Indications for ICI Therapy

\begin{tabular}{|c|c|c|}
\hline Ipilimumab; Yervoy $[4,9]$ & CTLA-4 & $\begin{array}{l}\text { Metastatic or unresectable melanoma, patients with cutaneous melanoma } \\
\text { including those with previous total lymphadenectomy }\end{array}$ \\
\hline Nivolumab; Opdivo $[4,9]$ & PD-1 & $\begin{array}{l}\text { Metastatic melanoma or unresectable BRAF V600 mutation-positive, } \\
\text { BRAF V600 wild-type unresectable melanoma, metastatic or unresectable } \\
\text { melanoma in combination with ipilimumab, metastatic non-small cell } \\
\text { lung cancer, advanced renal cell carcinoma, classic Hodgkin's lymphoma, } \\
\text { recurrent or metastatic squamous cell carcinoma of the head and neck } \\
\text { (HNSCC), metastatic urothelial carcinoma, hepatocellular carcinoma, } \\
\text { colorectal cancer with MSI-H and MMR aberrations }\end{array}$ \\
\hline $\begin{array}{l}\text { Atezolizumab; Tecentriq } \\
\qquad[4,9]\end{array}$ & PD-L1 & Metastatic urothelial carcinoma, metastatic NSCLC \\
\hline Avelumab; Bavencio [9] & PD-L1 & Merkel cell carcinoma, metastatic urothelial carcinoma \\
\hline Durvalumab; Imfinzi $[9,10]$ & PD-L1 & Metastatic urothelial carcinoma, unresectable stage III NSCLC \\
\hline
\end{tabular}

\section{References}

1. Schadendor $f D$, Hodi FS, Robert C, et al. Pooled Analysis of LongTerm Survival Data From Phase II and Phase III Trials of Ipilimumab in Unresectable or Metastatic Melanoma. Journal of clinical oncology : Official journal of the American Society of Clinical Oncology. 2015; 33: 1889-1894.

2. Remon J, Besse B, Soria JC. Successes and failures: what did we learn from recent first-line treatment immunotherapy trials in non-small cell lung cancer? BMC medicine. 2017; 15: 55.

3. Heinzerling L, Ott PA, Hodi FS, et al. Cardiotoxicity associated with CTLA4 and PD1 blocking immunotherapy. Journal for immunotherapy of cancer. 2016; 4: 50.

4. Jain V, Bahia J, Mohebtash M, Barac A. Cardiovascular Complications Associated With Novel Cancer Immunotherapies. Current treatment options in cardiovascular medicine. 2017; 19: 36.

5. Melero I, Hervas-Stubbs S, Glennie M, Pardoll DM, Chen L. Immunostimulatory monoclonal antibodies for cancer therapy. Nature reviews Cancer. 2007; 7: 95-106.

6. Varricchi G, Galdiero MR, Marone G, et al. Cardiotoxicity of immune checkpoint inhibitors. ESMO open. 2017; 2: e000247.

7. Hurst JH. Cancer immunotherapy innovator James Allison receives the 2015 Lasker DeBakey Clinical Medical Research Award. The Journal of clinical investigation. 2015; 125: 37323736.

8. Hodi FS, O'Day SJ, McDermott DF, et al. Improved survival with ipilimumab in patients with metastatic melanoma. The New England journal of medicine. 2010; 363: 711-723.
9. Rotte A, Jin JY, Lemaire V. Mechanistic overview of immune checkpoints to support the rational design of their combinations in cancer immunotherapy. Annals of oncology : official journal of the European Society for Medical Oncology. 2018; 29: 71-83.

10. FDA. Hematology/Oncology (Cancer) Approvals \& Safety Notifications. 2018.

11. Hodi FS, Chesney J, Pavlick AC, et al. Combined nivolumab and ipilimumab versus ipilimumab alone in patients with advanced melanoma: 2-year overall survival outcomes in a multicentre, randomised, controlled, phase 2 trial. The Lancet Oncology. 2016; 17: 1558-1568.

12. Postow MA, Sidlow R, Hellmann MD. Immune-Related Adverse Events Associated with Immune Checkpoint Blockade. The New England journal of medicine. 2018; 378: 158-168.

13. Johnson DB, Balko JM, Compton ML, et al. Fulminant Myocarditis with Combination Immune Checkpoint Blockade. The New England journal of medicine. 2016; 375: 1749-1755.

14. Geisler BP, Raad RA, Esaian D, Sharon E, Schwartz DR. Apical ballooning and cardiomyopathy in a melanoma patient treated with ipilimumab: a case of takotsubo-like syndrome. Journal for immunotherapy of cancer. 2015; 3: 4.

15. Reddy N, Moudgil R, Lopez-Mattei JC, et al. Progressive and Reversible Conduction Disease With Checkpoint Inhibitors. The Canadian journal of cardiology. 2017; 33: e1313-1315.

16. Brustle K, Heidecker B. Checkpoint inhibitor induced cardiotoxicity: managing the drawbacks of our newest agents against cancer. Oncotarget. 2017; 8: 106165-106166. 
17. Wang DY, Okoye GD, Neilan TG, Johnson DB, Moslehi JJ. Cardiovascular Toxicities Associated with Cancer Immunotherapies. Current cardiology reports. 2017; 19: 21.

18. Tajmir-Riahi A, Bergmann T, Schmid M, Agaimy A, Schuler G, Heinzerling L. Life-threatening Autoimmune Cardiomyopathy Reproducibly Induced in a Patient by Checkpoint Inhibitor Therapy. Journal of immunotherapy (Hagerstown, Md : 1997). 2018; 41: 35-38. 